\title{
Patient preferences of decision-making in the context of genetic testing for breast cancer risk
}

\author{
Almut W. Helmes, $M S^{1,2}$, Deborah J. Bowen, $P h D^{1}$, and Jürgen Bengel, $P h D, M D^{2}$
}

\begin{abstract}
Purpose: We conducted a study on women's preferred physician involvement in the decision to obtain genetic testing for breast cancer risk. Methods: Women from a primary care physician network completed a telephone survey and a written baseline questionnaire. Results: The majority preferred to make up their own minds. Predictors of leaving the decision to providers were less education, less knowledge, and higher external health locus of control. Trust in one's physician mediated the effect of health locus of control on decision method. Conclusion: Physicians will face requests from low-risk women and will need effective communication skills to inform women about the test. Genet Med 2002:4(3):150-157.
\end{abstract}

Key Words: genetic testing, breast cancer, decision-making, autonomy, physician

In the traditional physician-patient relationship, the patient's primary obligations are to actively seek professional help when needed and then to cooperate with the physician's decisions and comply with them. ${ }^{1}$ Arguments in favor of this paternalistic approach are based on the belief that physicians are experts in the field of health and illness and are, therefore, able to make the best decisions for the patient. The inherent inequality of knowledge between the physician and the patient is the motivation for this type of decision-making. It may also be too time consuming to present the patient with enough information to close this gap. It is also argued that it causes a patient an unnecessary amount of burden to be exposed to information they cannot comprehend. ${ }^{2,3}$

Since the 1960s, there has been a movement toward increased patient autonomy and involvement in decision-making. As a result, the concept of "informed consent" was recognized as a patient right in 1971. ${ }^{1,4}$ Patients are included in the decision-making process because they are increasingly informed and able to voice their own opinions about treatment options. ${ }^{5,6}$ Also, with medical technology continuously advancing, health care choices become more complicated and patient preferences become more important. Respect for autonomy has become a fundamental moral principle in bioethics, and it is argued that the physician's respect for the patient's autonomy is an integral part of her or his customary professional behavior. ${ }^{7-9}$ Physicians can benefit by sharing medical decision-making, in that they are not solely responsible for the decisions made, which may result in a decrease in malpractice suits. ${ }^{10,11}$ Patients may benefit by feeling more in control over their lives in the face of an illness.

From the ${ }^{1}$ Fred Hutchinson Cancer Research Center, Seattle, Washington; and ${ }^{2}$ University of Freiburg, Freiburg, Germany.

Deborah J. Bowen, PhD, Fred Hutchinson Cancer Research Center, 1100 Fairview Avenue North, MP900, Seattle, WA 98109.

Received: December 13, 2001.

Accepted: February 22, 2002.
There are few studies investigating which form of medical decision-making is preferred or superior. Some studies state that more patient autonomy may lead to better clinical outcomes, more compliance, and more patient satisfaction with clinical practice, ${ }^{1,12-15}$ but few of these are empirical studies. A study by Schulman found that patients who are active participants in the treatment process are significantly more likely to have their blood pressure under control. ${ }^{16}$ Another study found less discomfort, greater alleviation of symptoms, and more improvement in medical conditions in "active" patients compared with "passive" patients. ${ }^{17}$ Patients also expressed more control over their illness and more satisfaction with their physicians. Similar findings are reported by Lerman et al.: patient participation in decision-making and physician facilitation of patient involvement were significantly related to patients' satisfaction. ${ }^{18}$ Patient satisfaction appears to be one of the factors associated with compliance with therapeutic regimen, which in turn often determines the effectiveness of a treatment.

Patient demographic characteristics, such as age and education, influence the preference in decision-making. Younger and more educated patients often prefer an active role. ${ }^{19-21}$ More consumer-oriented individuals and those who are accustomed to making their own decisions in other settings are also more likely to participate in the decision process. Another factor is the severity of a disease, where there is evidence that a patient's desire to make decisions declined as he or she faced more severe illnesses. ${ }^{19}$

The nature of the medical decision to be made is also important. When different treatment options are similarly appropriate, physicians are more likely to elicit shared decision-making. When situations lack equipoise (e.g., in emergency care) or include conflict (e.g., where patient preferences are contrary to the physician's opinion), however, physicians are reluctant to share treatment decisions with patients. ${ }^{21}$ There is little research on decision methods in situations for which medical data are unavailable, unknown, or ambiguous. It may be that 
exposing uncertainties causes anxiety to both the clinician and the patient. One can argue that the more ambiguous a medical procedure (i.e., risks and benefits are uncertain), the more the patient may want guidance from the physician. This may be especially true for patients who tend to see their health controlled by powerful others. ${ }^{22}$ It may also be important in the trust patients have in their physicians or the knowledge patients have about a procedure.

Genetic testing for breast cancer risk is a procedure for which risks and benefits are currently uncertain and debated. Potential benefits are the relief of knowing that one is not a carrier after a negative test result, and the possibility of increasing screening behavior or obtaining interventions like chemoprevention or prophylactic mastectomy after a positive test result. There are also potential risks. Some women may decrease their screening behavior after a negative test result, taking the result as a "guarantee" that they will not develop breast cancer. ${ }^{23}$ Many women will receive inconclusive test results, rather than gaining the new and clear information for which they had hoped. ${ }^{24,25}$ This inconclusive result will especially occur for those who do not have affected relatives who can be tested first. Family dynamics may change in the face of genetic testing. ${ }^{26}$ Some women may feel pressured to get tested, although they do not want the test themselves, whereas others may experience "survivor guilt."

Despite these limitations, many studies show that women (even women at low risk for breast cancer) are interested in obtaining a genetic test for breast cancer risk. ${ }^{27-29}$ Women with a first degree relative (FDR) with breast cancer also propose free access to the test. A study by Audrain et al. reported that the majority of women indicated that they should be able to self-refer for genetic testing without needing a referral from a physician. ${ }^{30}$ Another study found that $95 \%$ of women with a family history of breast cancer agree that a person should be able to have genetic testing against a doctor's recommendation. ${ }^{31}$ There are no similar studies on women at low risk for breast cancer.

The purpose of this study was to elucidate women's preferences in decision-making in the context of genetic testing for breast cancer, using a population-based sample. How much physician involvement do women want in the decision to get tested? What are predictors of choosing one decision-making method over the other? Do demographic background variables like age or education play a role? Does a general tendency to believe that powerful others are in control over one's health influence decision-making methods in the context of genetic testing for breast cancer risk? If yes, then is this less the case for women who already have some knowledge on the subject or is it not the health locus of control but the trust in the physician that determines the decision-making method?

\section{MATERIALS AND METHODS}

\section{Participants}

Three hundred forty women were recruited through the largest primary care physician network in Washington state.
Women were eligible for the study if they were between 18 and 64 years old, had no personal history of breast or ovarian cancer, had not previously obtained genetic testing for cancer risk, lived in the Seattle/King County area (and planned to live there for the next year), were a patient at the network, were covered by a commercial health plan (not Medicare or Medicaid), and expressed the willingness and gave consent to be a participant in the randomized trial. The study was approved by the Fred Hutchinson Cancer Research Center Institutional Review Board.

\section{Procedures}

From the physician network, we selected two regions containing 10 clinics and received a list of all managed care patients who had a paid claim within the last year to get contact information that was most up to date. We sent each randomly selected potential participant a letter briefly describing the study $(N=4,690)$. The letter requested the woman's permission to contact her by phone and provided a number to call to take her name off the contact list ( $N=65$ called). Study staff called the remaining women for whom we had a valid address $(N=$ 3,933 ) and asked them to complete a brief telephone survey consisting of eligibility criteria, demographic information, and questions on perceived risk, cancer worries, and interest in genetic testing for breast cancer. All eligible participants then received a questionnaire packet in the mail $(N=919)$. Women were asked to return the completed baseline survey and signed consent form in a postage-paid envelope within 2 weeks $(N=$ $340)$. The recruitment process is described in more detail in Helmes et al. ${ }^{32}$

\section{Measures}

The following variables were assessed in the baseline survey:

\section{Demographic variables}

We measured demographic variables using simple singleitem measures. Ethnicity, marital status, education, employment, and household income were measured by asking participants to choose from categories (see Table 1). Participants were also asked to write in their current age and the number of people supported by the income stated. Number of FDR was measured by collecting a complete pedigree of family cancer history, including all generations in memory. We asked the cancer family history of every family member, living or deceased, including the type of cancer, age of onset, whether it was the first or second cancer, and mortality.

\section{Decision-making method}

In the section on genetic testing for breast cancer risk, three items were used to measure how women prefer to make decisions about this procedure. Items were phrased, "I prefer to leave all decisions regarding medical tests to my provider" (Low Autonomy); "I prefer to make decisions about medical tests myself, after considering my provider's opinion" (Mixed Autonomy); and "If the risks and benefits are uncertain regarding a medical procedure, I'd rather my 
Table 1

Demographics $(N=340)$

Mean age (SD)

$40.7(9.7)$

Ethnicity (\%)

White, non-Hispanic

85

White, Hispanic

3

Black or African American

4

Asian or Pacific Islander

American Indian, Aleut or Eskimo

Other

Marital status (\%)

Single

Married

Living with a partner

Widowed

Separated

Divorced

Education (\%)

Kindergarten through 8th grade

0

9th through 11th grade

High school graduate or GED

Post high school training or some college

Graduated from college

Graduate or professional school

11

1

2

9

Employment (\%)

Full-time employed

66

Part-time employed

20

Not employed (incl. student or homemaker)

Retired

Household income (\%)

Less than $\$ 15,000$

$\$ 15,000$ to $\$ 29,999$

$\$ 30,000$ to $\$ 49,999$

23

$\$ 50,000$ to $\$ 69,000$

25

$\$ 70,000$ or over

Mean no. of people supported by income (SD)

No. of FDR with breast cancer (\%)

0

1

85

14

2

physician told me that and let me make up my own mind" (Ambiguous Autonomy). Answer choices were "strongly disagree" (1), "somewhat disagree" (2), "somewhat agree" (3), and "strongly agree" (4).

\section{Health locus of control}

We measured health locus of control (HLOC) with a scale developed by Wallston et al. ${ }^{33}$ The scale measures three dimensions of HLOC beliefs: internal, powerful others, and chance. The scale includes six items for each dimension and scores on a 6-point scale from 1 (strongly disagree) to 6 (strongly agree). In this study we included the powerful others dimension (HLOC PO). The reported Cronbach $\alpha^{\star}$ of the scale was 0.67.

\section{Knowledge on breast cancer genetics and BRCA1 testing}

We assessed knowledge about genetics and testing by asking 11 true or false questions according to Lerman at al. ${ }^{34}$ Items included, "A father can pass down an altered BRCA1 gene to his children" and "One half of breast cancer cases occur in women who have an altered BRCA1 gene." One point was given for each correct answer. The Cronbach $\alpha$ was 0.77.

\section{Trust in provider}

Participants' attitudes toward their primary care provider were measured using a scale (unpublished) developed as part of a core set of instruments for a consortium of the National Institutes of Health-funded genetic testing projects (Cancer Genetic Studies Consortium). The scale is scored on a 4-point scale from 1 (strongly disagree) to 4 (strongly agree). The subscale Trust/Satisfaction has five items, including, "I feel I can trust my doctor's judgments" and "I am satisfied with my current health care provider." The Cronbach $\alpha$ of the scale was 0.85 .

\section{Statistical analysis}

We analyzed the data in three steps. First, we examined the frequency distribution of women's preferences in physician involvement in medical decision-making. As a second step in the analysis, we looked at different predictors of choosing a decision method. Different sets of regression analyses were used to test the predictive value of two background variables (age and education) and three psychological variables (HLOC $\mathrm{PO}$, knowledge about genetics and testing and trust in provider) for the three decision-making methods. We used linear regression to test whether knowledge has a moderating effect on decision-making. A moderator is a variable that affects the relation between a predictor variable and a dependent variable. ${ }^{35}$ To test the effect of the moderator, the interaction or product of the moderator and the independent variable is included in the regression equation, and if it proves to be significant, there is evidence for a moderator effect. ${ }^{36}$ Third, we tested the mediating effect of trust in one's physician on decision-making again using linear regression. A mediator is a variable that accounts for the relation between the predictor and the dependent variable. ${ }^{35}$ We estimated three equations: regressing the mediator on the independent variable, regressing the dependent on the independent variable, and regressing the dependent variable on both the independent variable and the mediator. If all equations prove the effect in the predicted di-

\footnotetext{
${ }^{*}$ The Cronbach $\alpha$ is the index of internal consistency of a test.
} 
rection and the effect of the independent on the dependent variable is less in the third equation than in the second, a mediator effect is present.

\section{RESULTS}

\section{Study sample}

The description of the study sample is given in Table 1 . Women in our sample were predominantly white (85\%). For most ethnic groups, the distribution came close to the King County statistical profile of 1998 , where $80 \%$ were white, $3 \%$ Nonwhite Hispanic, 5\% African American, 10\% Asian or Pacific Islander, and 1\% American Indian. ${ }^{37}$ The average age of our study sample was 40.7 years with a range of 19 to 64 and the majority was married or living with a partner (70\%). Sixty-six percent of the women had a college degree or higher and the majority (86\%) were employed either full- or part-time. With many married women and likely both partners working, it is no surprise that combined household income was fairly high, supporting an average of 2.5 individuals per household. The majority of the women in the sample had no FDR with breast cancer (85\%), 14\% had one FDR with breast cancer, and 1\% had two.

\section{Decision-making methods}

Women's preferences of physician involvement in medical decision-making concerning genetic testing for breast cancer risk are presented in Table 2. Our research questions stated different levels of patient involvement in decision-making. Very few women $(7 \%)$ strongly agreed that they would prefer to leave all decisions regarding medical tests to their provider, and more than half the women disagreed with this statement (59\%). Most women (86\%) either somewhat or strongly agreed with the statement to prefer to make decisions about medical tests themselves, after considering their provider's opinion. Seventy-seven percent of all women strongly agreed that, when risks and benefits are uncertain regarding a medical procedure, they would prefer that their physicians would inform them of the risks and benefits and then let them make up their own mind.

\section{Table 2}

Women's preferences of physician involvement in medical decision-making $(N=340)$

\begin{tabular}{lcccc}
\hline & $\begin{array}{c}\text { Strongly } \\
\text { disagree }\end{array}$ & $\begin{array}{c}\text { Somewhat } \\
\text { disagree }\end{array}$ & $\begin{array}{c}\text { Somewhat } \\
\text { agree }\end{array}$ & $\begin{array}{c}\text { Strongly } \\
\text { agree }\end{array}$ \\
\hline Leave decisions to my provider & 24 & 35 & 34 & 7 \\
$\begin{array}{l}\text { Make decisions myself after } \\
\text { considering providers } \\
\text { opinion }\end{array}$ & 4 & 9 & 47 & 40 \\
$\begin{array}{l}\text { When risks and benefits } \\
\text { uncertain want to make } \\
\text { up my own mind }\end{array}$ & 2 & 3 & 18 & 77 \\
\hline
\end{tabular}

Values represent percentages.

\section{Predictors of preferred decision-making method}

First, we tested the effect of the background variables (age and education), HLOC PO, and knowledge in predicting the three different decision-making methods. Table 3 contains the results of those regression analyses.

In the background variables, education was a significant predictor for the decision-making method of Low Autonomy but not for Mixed or Ambiguous Autonomy. Age was not a significant predictor for any of the three methods. The background variable set contributed only $2 \%$ of the variance to Low Autonomy and virtually none to the other two decision-making methods. The second set of variables entered into the regression increased the explained variance for Low Autonomy to $15 \%$. Both HLOC PO and knowledge were significant predictors of this set. We also entered the interaction of knowledge and HLOC PO into the equation. It was nonsignificant, thereby showing no moderator effect. For the other two decision methods, neither HLOC PO knowledge nor the product of the two were significant predictors and the explained variances only marginally increased by entering this set into the equations.

To test the hypothesis that trust in physicians has a mediating effect on the relationship of HLOC PO and decision-making method, we performed three regressions (see Table 4). The first regression tested the effect of the independent variable (HLOC PO) on the mediator (trust). As seen in the first equation of Table 4 there was a significant $(P=0.036)$ positive relationship for this equation. The second regression tested the effect of the independent variable on the dependent variables (each of three decision-making methods). For the dependent variable Low Autonomy, this was highly significant $(P=$ $0.000)$ in a positive direction. For Ambiguous Autonomy, it was of marginal significance $(P=0.061)$ in a negative direction. For Mixed Autonomy, there was no effect of the independent on the dependent variable. In the third regression, we tested whether or not the mediator affected the dependent variable. This was true for the first decision method of Low Autonomy, for which there was a highly significant positive relationship $(P=0.000)$. Again, it was only marginally significant for Ambiguous Autonomy $(P=0.070)$ and not at all significant for the second decision method of Mixed Autonomy. For Ambiguous Autonomy, HLOC PO was now a significant predictor $(P=0.038)$, because the variable trust took up some of the standard error of the variable HLOC P. O. Women who wanted to make up their mind in ambiguous situations scored lower on the HLOC PO scale.

There was a mediator effect of trust in physicians on the relationship between HLOC PO and Low Autonomy, which can be seen in the $\beta$ value $\dagger$ of 0.318 for the third equation that was lower than 0.352 for the second equation. There was no mediator effect of trust for the decision method of Ambiguous Autonomy or for Mixed Autonomy, because adding trust to the equation did not lower the $\beta$ value for HLOC PO from the second to the third equation. The background variables of age

tThe $\boldsymbol{\beta}$ value is the standardized regression coefficient. 
Table 3

Moderator effect of knowledge on preferred decision-making

\begin{tabular}{|c|c|c|c|c|c|c|c|c|c|}
\hline & \multicolumn{3}{|c|}{ Low autonomy } & \multicolumn{3}{|c|}{ Mixed autonomy } & \multicolumn{3}{|c|}{ Ambiguous autonomy } \\
\hline & $B$ & $T$ & Sig. & $B$ & $T$ & Sig. & $B$ & $T$ & Sig. \\
\hline \multicolumn{10}{|l|}{ Background } \\
\hline Age & -0.013 & -0.254 & 0.799 & 0.088 & 1.595 & 0.112 & -0.012 & -0.220 & 0.826 \\
\hline \multirow[t]{2}{*}{ Education } & -0.101 & -1.952 & 0.052 & -0.008 & -0.135 & 0.893 & 0.040 & 0.722 & 0.471 \\
\hline & \multicolumn{3}{|c|}{$F(2,332)=2.89 ; P=0.057 ; R^{2}=0.017$} & \multicolumn{3}{|c|}{$F(2,331)=1.16 ; P=0.315 ; R^{2}=0.007$} & \multicolumn{3}{|c|}{$F(2,332)=0.67 ; P=0.512 ; R^{2}=0.004$} \\
\hline \multicolumn{10}{|l|}{ Predictors } \\
\hline HLOC PO & 0.255 & 3.373 & 0.001 & -0.075 & -0.924 & 0.356 & -0.147 & -1.814 & 0.071 \\
\hline Knowledge & -0.339 & -2.045 & 0.042 & -0.207 & -1.157 & 0.248 & -0.085 & -0.478 & 0.633 \\
\hline \multirow[t]{2}{*}{ HLOC PO $\times$ knowledge } & 0.292 & 1.690 & 0.092 & 0.229 & 1.227 & 0.221 & 0.143 & 0.768 & 0.443 \\
\hline & \multicolumn{3}{|c|}{$F(5,329)=11.83 ; P=0.000 ; R^{2}=0.152$} & \multicolumn{3}{|c|}{$F(5,328)=0.76 ; P=0.577 ; R^{2}=0.011$} & \multicolumn{3}{|c|}{$F(5,329)=1.22 ; P=0.297 ; R^{2}=0.018$} \\
\hline
\end{tabular}

Table 4

Mediator effect of trust in physician on preferred decision-making

Regression 1: Independent $\rightarrow$ Mediator

\begin{tabular}{lccc}
\hline & \multicolumn{2}{c}{ Trust } \\
\cline { 2 - 4 } & $B$ & $T$ & \multicolumn{1}{c}{ Sig. } \\
\hline Age & 0.021 & 0.375 & 0.708 \\
Education & 0.048 & 0.881 & 0.379 \\
HLOC PO & 0.115 & 2.105 & 0.036 \\
& & $F(3,331)=1.72 ; P=0.162 ; R^{2}=0.015$ \\
\hline
\end{tabular}

Regression 2: Independent $\rightarrow$ Dependent

\begin{tabular}{|c|c|c|c|c|c|c|c|c|c|}
\hline & \multicolumn{3}{|c|}{ Low autonomy } & \multicolumn{3}{|c|}{ Mixed autonomy } & \multicolumn{3}{|c|}{ Ambiguous autonomy } \\
\hline & $B$ & $T$ & Sig. & $B$ & $T$ & Sig. & $B$ & $T$ & Sig. \\
\hline Age & -0.017 & -0.334 & 0.739 & 0.083 & 1.513 & 0.131 & -0.016 & -0.289 & 0.773 \\
\hline Education & -0.107 & -2.098 & 0.037 & -0.002 & -0.044 & 0.965 & 0.052 & 0.940 & 0.348 \\
\hline \multirow[t]{2}{*}{ HLOC PO } & 0.352 & 6.872 & 0.000 & -0.002 & -0.030 & 0.976 & -0.103 & -1.881 & 0.061 \\
\hline & \multicolumn{3}{|c|}{$F(3,331)=17.94 ; P=0.000 ; R^{2}=0.140$} & \multicolumn{3}{|c|}{$F(3,330)=0.77 ; P=0.511 ; R^{2}=0.007$} & \multicolumn{3}{|c|}{$F(3,331)=1.63 ; P=0.182 ; R^{2}=0.015$} \\
\hline
\end{tabular}

Regression 3: Mediator, Independent $\rightarrow$ Dependent

\begin{tabular}{|c|c|c|c|c|c|c|c|c|c|}
\hline & \multicolumn{3}{|c|}{ Low autonomy } & \multicolumn{3}{|c|}{ Mixed autonomy } & \multicolumn{3}{|c|}{ Ambiguous autonomy } \\
\hline & $B$ & $T$ & Sig. & $B$ & $T$ & Sig. & $B$ & $T$ & Sig. \\
\hline Age & -0.025 & -0.508 & 0.612 & 0.083 & 1.504 & 0.134 & -0.018 & -0.327 & 0.744 \\
\hline Education & -0.124 & -2.529 & 0.012 & -0.002 & -0.028 & 0.978 & 0.047 & 0.854 & 0.394 \\
\hline Trust & 0.292 & 5.977 & 0.000 & 0.003 & 0.055 & 0.956 & 0.100 & 1.820 & 0.070 \\
\hline HLOC & 0.318 & 6.486 & 0.000 & -0.039 & -0.700 & 0.484 & -0.115 & -2.084 & 0.038 \\
\hline
\end{tabular}

$\mathrm{PO}$

and education only explained up to $2 \%$ of the variance in any of the regressions; therefore, $R^{2}$ values were not presented separately. Education was a significant predictor of the decisionmaking method of Low Autonomy for the second and third equation, whereas age was not significant.

\section{DISCUSSION}

Data from this study support the hypothesis that many patients want to be autonomous in their medical decision-making concerning genetic testing for breast cancer risk. Although 
some patients still preferred to leave all medical decisions to their provider, the majority of participants wanted to take their provider's opinion into account but then make the decisions themselves. When there was an element of ambiguity, almost all women stated that they would want to make up their own mind. This finding is of special interest in the context of genetic testing for breast cancer risk, for which most women in the general population, many physicians, and even some experts are undecided about possible risks and benefits of this procedure. If many women at low risk are interested in the test ${ }^{27-29}$ and they want to make up their own mind about obtaining the test, one can expect that requests for this test will increase, independent of physician recommendation. The role of the physician then is to critically examine the need for genetic testing. ${ }^{38}$ If a physician finds that an individual is not an appropriate candidate for genetic testing, thereby opposing the patient's will, then effective communication skills are essential. A paternalistic physician who states only that genetic testing is not an option may provoke some patients who are interested in the test and burden the physician-patient relationship. However, a physician who introduces shared decision-making will inform the patient about the limitations of the test, elicit the patient's reasons for wanting the test, and discuss possible misconceptions about the test. ${ }^{39}$

Different factors may play a role in who prefers which decision-making method. Our data confirmed findings in the literature $^{19-21}$ in that education significantly correlated with women's preference to leave the decision to their providers; women with more education were less likely to agree to let their provider make decisions about medical tests than those with less education. Age was not a significant correlate for the decision-making method. A possible explanation for this could be related to the fact that our study sample was limited to women age 18 through 64 and did not include any older women. The movement toward more patient autonomy has been ongoing for approximately 40 years, and most women of our sample were still young during the beginning of this movement and may have adapted to the new decision-making method.

Other factors that may play a role in medical decision-making are the degree to which patients see their health under the control of powerful others, the knowledge about a certain medical procedure, and a patient's trust in the physician. HLOC PO was a significant predictor of women's preference to leave medical decisions to their provider. The higher women scored on the HLOC PO scale the more they preferred leaving the decision to the provider. HLOC PO did not prove to influence the other two decision-making methods. This finding could be because answers to these two questions showed a ceiling effect. Most participants chose the same or similar answers, thus limiting the variance of these items.

The amount of knowledge about genetic testing for breast cancer risk and testing by was also a significant predictor of women's preference to leave medical decisions to their provider. Women with less knowledge about the test were more likely to choose this decision-making method. We had expected, however, that more knowledge about the test would also correlate with an increased preference for patient involvement in the other two decision-making methods, especially in ambiguous situations. This did not hold true and could again be due to the ceiling effect. We also tested the hypothesis that knowledge had a moderator effect on the decision-making method. A moderator effect would indicate that women who score highly on the HLOC PO scale prefer to leave medical decisions to their provider, but less so if they have extensive knowledge about a procedure. Our data did not support this hypothesis in that the moderator effect was not significant.

These data show that women do not base their decisionmaking method in ambiguous situations on the amount of knowledge they have about a medical procedure. Women in our study had low knowledge about breast cancer risk and testing. This finding is in concordance with data from other studies. ${ }^{34,40,41}$ At the same time, many indicated that they prefer to make up their own mind about whether to obtain genetic testing for breast cancer risk. This finding adds another argument for sharing information and decision-making with patients.

Genetic counselors can assist with decisions about genetic testing. The goal of genetic counseling in the context of genetic testing for breast cancer is to inform the patient about the risks and benefits of the test and to put it in perspective for the individual. For example, a woman without a family history of breast cancer will learn that there is very little she can gain from taking the test. This modifies the concept of "nondirectiveness" of genetic counseling. In many situations, genetic counselors are said to espouse a nondirective method (e.g., leaving the decision whether to abort a fetus with down-syndrome or malformations to the parents). ${ }^{42}$ In the context of genetic counseling for breast cancer risk, there is no reason to limit the counselor from making recommendations about a course of action, if a woman is not an appropriate candidate based on her family history. A partnership among a patient, a physician, and a genetic counselor could provide an appropriate and supportive situation for these important decisions.

We hypothesized that trust in one's physician could account for the relation between HLOC PO and the decision-making method. The question of whether to involve physicians in decision-making processes may not depend on the perspective that powerful others are in control of one's health but on the trust they have for their physicians' judgment. This mediator effect of trust in physicians on the relationship between HLOC $\mathrm{PO}$ and the preference to leave medical decisions to the provider proved to be significant; however, it was small, and HLOC PO still was the most significant predictor of the equation after trust had been entered. Therefore, both HLOC PO and trust were important correlates of preferring to leave medical decisions to the provider. Trust was not related to the other two decision-making methods (Mixed Autonomy and Ambiguous Autonomy). This finding rules out the hypothesis that women want to make their own decision in medical care because they do not trust their physicians.

There are some limitations of the present study. We used the term "medical test" in two of the questions and "medical pro- 
cedure" in the third. Because the terms were used in the section on genetic testing and followed each other, we do not expect that the terms were interpreted differently. Most women marked similar answer choices for the two decision-making methods of mixed autonomy and ambiguous autonomy. The limited variance restricts the use of these items as dependent variables to some degree; however, ambiguous autonomy had the least variance but still showed some marginally significant results; therefore, we decided to include them in analyses. Regression analyses did not allow any interpretation of causality; therefore, we cannot tell whether these women feel comfortable leaving their health in the hands of powerful others because they have trust in their physicians or whether they have trust in their physicians because they prefer to leave medical decisions to powerful others, thereby needing the trust as a means of dissonance reduction. ${ }^{43}$ Finally, these data cannot be generalized to women from lower socioeconomic backgrounds. Even within this data set, education changed the response so future research must focus on broader demographic groups.

\section{CONCLUSIONS}

The study showed that women from the general population prefer increased patient autonomy and involvement in decision-making over medical paternalism by the physician in the context of genetic testing for breast cancer risk. This finding has practice implications in that women will ask their primary care providers for a referral to testing rather than a recommendation whether testing is appropriate for them. Physicians will need to face these requests with effective communication skills, informing patients about the test, and pointing out possible misconceptions. Other roles could be to discuss pros and cons and to refer patients for genetic evaluation. Thereby they can fulfill their role as gatekeepers to the test and prevent low-risk women from taking the test who will have no benefit from it.

Not all patients have the desire to participate in medical decision-making. Some patients may feel more comfortable leaving their health in the hands of powerful others, i.e., their physicians and other health professionals who make the decisions and tell the patients how to best cooperate with treatment regimens. Patients who have this external locus of control should not be forced to be autonomous in their health care. In a functioning physician-patient relationship, the physician will offer the patient a choice of the amount of information conveyed and the level of participation in the decision-making process, promoting confidence in the relationship. Physicians can make referrals to appropriate team professionals, such as genetic counselors, with follow-up if necessary. Finally, given the current data, allowing the patient to choose her path in the wake of education and discussion seems the best choice for further treatment.

\section{Acknowledgments}

This research was supported by grants from the National Human Genome Research Institute and the National Cancer
Institute (HG/CA01190), the Landesgraduiertenförderung Baden-Württemberg, and the German Academic Exchange Service (DAAD).

\section{References}

1. Brody DS. The patient's role in clinical decision-making. Ann Intern Med 1980;93: $718-722$.

2. Savulescu J. Rational non-interventional paternalism: why doctors ought to make judgments of what is best for their patients. J Med Ethics 1995;21:327-331.

3. Weiss GB. Paternalism modernised. J Med Ethics 1985;11:184-187.

4. Coulter A. Paternalism or partnership? Patients have grown up - and there is no going back. BMJ 1999;319:719-720.

5. Balint J, Shelton W. Regaining the initiative. Forging a new model of the patientphysician relationship. JAMA 1996;275:887-891.

6. Gallagher SM. Paternalism in healthcare decision making. Ostomy Wound Manage 1998;44:22-25.

7. Secker B. The appearance of Kant's deontology in contemporary Kantianism: Concepts of patient autonomy in bioethics. J Med Philos 1999;24:43-66.

8. Guadagnoli E, Ward P. Patient participation in decision-making. Soc Sci Med 1998; 47:329-339.

9. Gordon HH. The doctor-patient relationship. J Med Philos 1983;8:243-255.

10. Montgomery K. Information is not enough: the place of statistics in the doctorpatient relationship. Cancer Treat Res 2000;102:13-21.

11. Elwyn G, Edwards A, Kinnersley P. Shared decision-making in primary care: The neglected second half of the equation. Br J Gen Pract 1999;49:477-482.

12. Peters RM. Matching physician practice style to patient informational issues and decision-making preferences. Arch Fam Med 1994;3:760-763.

13. Jensen PS. The doctor-patient relationship: headed for impasse or improvement? Ann Intern Med 1981;95:769-771.

14. Delbanco TL. Enriching the doctor-patient relationship by inviting the patient's perspective. Ann Intern Med 1992;116:414-418.

15. Schain WS. Patients' rights in decision making: the case for personalism versus paternalism in health care. Cancer 1980;46:1035-1041.

16. Schulman BA. Active patient orientation and outcomes in hypertensive treatment. Application of a socio-organizational model. Med Care 1979;17:267-280.

17. Brody DS, Miller SM, Lerman CE, Smith DG, Caputo GC. Patient perception of involvement in medical care: relationships to illness attitudes and outcomes. J Gen Intern Med 1989;4:506-511.

18. Lerman C, Brody DS, Caputo GC, Smith DG, Lazaro C, Wolfson HG. Patients' perceived involvement in care scale: relationship to attitudes about illness and medical care. J Gen Intern Med 1990;5:29-33.

19. Ende J, Kazis L, Ash A, Moskowitz MA. Measuring patients' desire for autonomy. J Gen Intern Med 1989;4:23-30.

20. Cassileth BR, Zupkis RV, Sutton-Smith K, March V. Information and participation preferences among cancer patients. Ann Intern Med 1980;92:832-836.

21. Elwyn G, Edwards A, Gwyn R, Grol R. Towards a feasible model for shared decision making: focus group study with general registrars. BMJ 1999;319:753-756.

22. Wallston KA, Wallston B. Who is responsible for your health? The construct of health locus of control. In: Sanders GS, Suls J, editors. Social psychology of health and illness. Hillsdale: Erlbaum, 1982:65-96.

23. Lerman C, Hughes, Croyle R, Main D, Durham C, Snyder C, Bonney A, Lynch JF, Narod SA, Lynch HT. Prophylactic surgery decisions and surveillance practices one year following BRCA1/2 testing. Prevent Med 2000;31:75-80.

24. Collins FS. BRCA1: lots of mutations, lots of dilemmas. N Engl J Med 1996;334:186188.

25. Eng C, Vijg J. Genetic testing: the problems and the promise. Nat Biotechnol 1997; 15:422-426.

26. Schneider KA. Genetic counseling for BRCA1/BRCA2 testing. Genet Test 1997;1:9198.

27. Andrykowski MA, Munn RK, Studts JL. Interest in learning of personal genetic risk for cancer: a general population survey. Prevent Med 1996;25:527-536.

28. Ulrich CM, Kristal AR, White E, Hunt JR, Durfy SJ, Potter JD. Genetic testing for cancer risk: a population survey on attitudes and intention. Commun Genet 1998;1: 213-222.

29. Durfy SJ, Bowen DJ, McTiernan A, Sporleder J, Burke W. Attitudes and interest in genetic testing for breast and ovarian cancer. Susceptibility in diverse groups of women in Western Washington. Cancer Epidemiol Biomarkers Prev 1999;8:369-375.

30. Audrain J, Rimer B, Cella D, Garber J, Peshkin BN, Ellis J, Schildkraut J, Stefanek M, Vogel V, Lerman C. Genetic counseling and testing for breast-ovarian cancer susceptibility: what do women want? J Clin Oncol 1998;16:133-138.

31. Benkendorf JL, Reutenaure JE, Hughes CA, Eads N, Willison J, Powers M, Lerman C. Patients' attitudes about autonomy and confidentiality in genetic testing for breast-ovarian cancer susceptibility. Am J Med Genet 1997;73:296-303. 
32. Helmes AW, Bowen DJ, Bowden R, Bengel J. Predictors of participation in genetic research in a primary care physician network. Cancer Epidemiol Biomarkers Prev 2000;9:1377-1379.

33. Wallston KA, Wallston BS, DeVellis R. Development of the Multidimentional Health Locus of Control (MHLC) Scales. Health Educ Monogr 1978;6:160-170.

34. Lerman C, Biesecker B, Benkendorf JL, Kerner J, Gomez-Caminero A, Hughes C, Reed MM. Controlled trial of pretest education approaches to enhance informed decision-making for BRCA1 gene testing. J Natl Cancer Inst 1997;89: $148-157$.

35. Baron RM, Kenny DA. The moderator-mediator variable distinction in social psychological research: conceptual, strategic, and statistical considerations. J Pers Soc Psychol 1986;51:1173-1182.

36. Cohen J, Cohen P. Applied multiple regression/correlation analysis for the behavioral sciences, 2nd ed. Hillsdale: Erlbaum, 1983.

37. King County Office of Budget, and Strategic Planning. Annual Growth Report: Statistical Profile King County. King County, WA: Office of Budget and Strategic Planning, 1998.
38. Moulton G. Surgeons have critical role in genetic testing decisions, medical, legal experts say. J Natl Cancer Inst 1998;90:804-805.

39. Lanken PN. The challenge of medical decision making. Balancing patient autonomy and physician responsibility. Am Rev Respir Dis 1992;145:253-254.

40. Bluman LG, Rimer BK, Berry DA, Borstelmann N, Iglehart JD, Regan K, Schildkraut J, Winer EP. Attitudes, knowledge, and risk perceptions of women with breast and/or ovarian cancer considering testing for BRCA1 and BRCA2. J Clin Oncol 1999;17:1040-1046.

41. Lerman C, Narod S, Schulman K, Hughes C, Gomez-Caminero A, Bonney G, Gold K, Trock B, Main D, Lynch J, Fulmore C, Snyder C, Lemon SJ, Conway T, Tonin P, Lenoir G, Lynch H. BRCA1 testing in families with hereditary breast-ovarian cancer: a prospective study of patient decision making and outcomes. JAMA 1996;275: 1885-1892.

42. Elwyn G, Gray J, Clarke A. Shared decision making and non-directiveness in genetic counselling. J Med Genet 2000;37:135-138.

43. Festinger L. A theory of cognitive dissonance. Stanford: Stanford University Press, 1957:291. 